\title{
Thermal transfer of Nanofluids based on carbon nanotubes/glycerol and study of their rheological behavior
}

\author{
S. Lahlou ${ }^{1}$, R. Sehaqui ${ }^{1}$, N. Lahlou ${ }^{1}$ \\ ${ }^{1}$ Department of Physics, Faculty of Sciences Ain Chock, Hassan II University, 20470 Casablanca, Morocco \\ sara.lahlou20@gmail.com
}

\begin{abstract}
In the field of heat transfer, the use of the properties of rheological behavior inherent to suspensions of nanoparticles in heat transfer fluids has been the subject of several years of work. Our study concerns the particular case of suspensions of multilayered carbon nanotubes (MNTCs) in glycerol. We highlight the effect of the mass fraction $\varphi$ of (MNTCs) on the rheology of the solutions for $0.1 \% \leq \varphi \leq 1 \%$, on the temperature $\left[30-80^{\circ} \mathrm{C}\right]$ and the effect on a heat exchanger. The experimental results obtained are described by viscoplastic models for which the yield stress increases with $\varphi$. We then propose a law of polynomial evolution of the apparent viscosity as a function of the volume fraction for each value of the shear rate varying from 0 s- 1 to 100 s-1.
\end{abstract}

Keywords: Rheology, Nanofluid, carbon nanotube, empirical law, heat exchanger

\section{Introduction}

There is a real demand in the industrial world to develop new strategies to improve the thermal behavior of fluids used in heat exchangers. Significant progress has made it possible, since the end of the 1990s, to synthesise nanoscale particles, which, dispersed in a carrier liquid, constitute nanofluids. Their synthesis meets the need to improve the thermal properties by inserting a solid phase of very high thermal conductivity.

Based on previous studies of the rheological and thermal behavior of the suspensions, the effect of the volume fraction on the viscosity and temperature of the suspension was mainly emphasized in most studies, if not Virtually all as a Newtonian behavior, L. Chen [1] found that the behavior of the carbon nanotube with glycerol as the base fluid is Newtonian for different volume fractions. Other work of MBMoghaddam [5] is carried out on the rheological properties of graphemes (It is a carbon sheet) dispersed in glycerol which is described by the Newtonian behavior for different mass fractions up to 0.02 and different temperature and S. Halelfadf [2] found that the rheological behavior of nanofluids is Newtonian regardless of the applied shear rate. Our study is part of this framework and sets itself for essential objectives, the determination of the methods of elaboration of the solutions of carbon nanotubes dispersed in glycerol as well as the different measurement protocols for the analysis of the rheological and thermal behavior of suspensions. ,

We are interested in the impact of the volume fraction of the multilayer carbon nanotubes suspended in glycerol on the rheological behavior, the temperature and the influence in a heat exchanger, thus the connection, in the case of the suspensions at $F_{M}$ greater than 0.5 between the values of the apparent viscosities for given shear rates and the viscosity values of these suspensions deduced from the empirical formula. In this framework, we propose a polynomial-type relation, thus highlighting the viscoelasticity and determining the evolution of the critical frequency (point of intersection of the loss and conservation modulus) as a function of the concentration.

\section{Raw materials and materials: \\ 2.1 Raw materials}

For the development of nanofluids tested in this work, we consider the following products:

Multilayer carbon nanotube (purity> 95\%)

Glycerol, liquid chosen as a heat transfer medium in which the nanoparticles can remain in stable suspension thanks to its high viscosity.

\subsection{Protocol of elaboration:}

The nanofluid obtained from the following steps:

- Addition of the carbon nanotube powder in glycerol while continuing to heat and stir in the presence of a magnetic stirring of 10 revolutions / sec for 1 hour according to the different fractions desired volume: [0.1\% $-1 \%]$ and the different temperatures $\left[30-80^{\circ} \mathrm{C}\right]$.

The suspension is subjected to magnetic stirring using a thermostated bath of carbon nanotubes dispersed in glycerol.

\subsection{Materials and methods:}

-For the rheological characterization:

The rheological tests are conducted as part of this study using a HAAKE RHEOSTRESS 1 rotary rheometer with a plane-plane geometry, for the tests carried out as part of this work, the configurations are selected are: a quasistationary configuration for monitoring the rheological behavior of the nanofluid, an oscillatory configuration for determining the critical frequency.

-For thermal characterization:

The thermal tests are conducted using a double jacket heat exchanger to detect the heat gain.

\section{Results and discussion:}

\subsection{Rheological behavior:}

\section{Effect of the volume fraction [0.1\% -1\%]:}

Two types of flow regime are retained:

\section{- In quasi-stationary mode:}

We initially focused on the evolution of shear stress and viscosity as a function of shear rate. We will take from RheoStress 1 a set of rheograms which allow to highlight the effect of the concentration on apparent viscosity. 


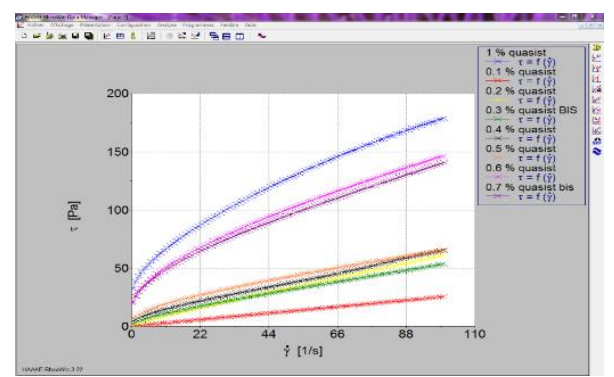

Fig. 1. The shear stress versus nanofluid shear rate for different Fraction

From the measurement of the rheological properties of carbon nanotubes-glycerol, we can draw the following conclusions: - According to the rheogram which relates the shear stress as a function of the shear rate of the different mass fractions, we notice that there are three distinct zones such that the first one presents the Newtonian behavior of $0.1 \%$ and $[0.2 \%, 0.3 \%, 0.4 \%$, $0.5 \%$ ] behavior of Herschel Bulkley with a low threshold constraint and of $[0.6 \%, 1 \%]$ a Herschel Bulkley behavior with a remarkable stress-threshold, where the threshold stresses are respectively equal to 0.9959 up to 27.54 $\mathrm{Pa}$

-According to the underside curve, the effect of the mass fraction strongly affects the threshold stress, the consistency and the flow index, and the graphs show that the threshold stress and the consistency increase with increase of the fraction. On the other hand, the index of the flow decreases

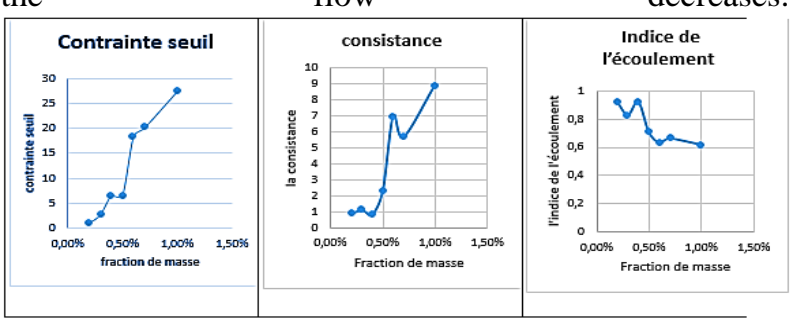

Fig. 2. The rheological parameters of the nanofluid

Published empirical research on suspended particles has focused on determining the relationship between apparent viscosity and particle volume fraction.

Several theoretical models have been developed to predict the evolution of the dynamic viscosity of suspensions under certain conditions. In this part, we will focus on the theoretical models most adapted to our different Mass Fractions.

Law of evolution of the apparent viscosity as a function of the volume fraction for each value of the shear rate varying from $10 \mathrm{~s}-1$ to $80 \mathrm{~s}-1$.

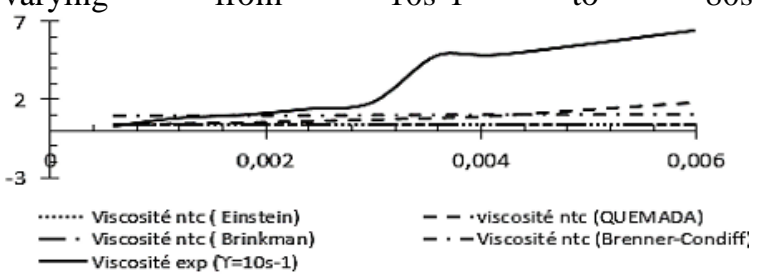

Figure. 3: Estimation of the relative macroscopic viscosity as a function of the particulate volume fraction for the suspensions of carbon nanotubes.

The model closest to our case in different shear rates is the model of QUEMADA according to theoretical calculations for volume fractions ranging from 0.0006 to 0.003 .

Beyond 0.003 to 0.006 , there are no theoretical models that quantify the non-Newtonian behavior, so we have drawn from the experimental results the established relation (1) which describes the law is the polynomial law of order2 is given as follows:

$\eta=\mathrm{a} \varphi \mathrm{v} 2+\mathrm{b} \varphi \mathrm{v}+\mathrm{c}$

The coefficients of the polynomial were determined by the least squares method and removed by the experimental results; we take: $\sigma=\mathrm{b} / \mathrm{a} \approx-0.01$ and $\beta=\mathrm{c} / \mathrm{a} \approx 2 \mathrm{E}-05$

So: the polynomial law of order 2 for the fractions volumetric beyond 0.003 established is:

$$
\eta=a(\Upsilon)\left(\varphi \vee 2-0.01^{*} \varphi v+2\right. \text { E-05) }
$$

Hence: $\varphi \mathrm{V}$ : Volume fraction, $\eta$ : Viscosity

a: Coefficient of the order polynomial (2)

With the help of this expression, one comes to consolidate the protocols in quasi-stationary mode on the one hand and to optimize the processes of their use in industrial environment on the other hand.

\section{- In oscillating mode:}

For this regime, we studied the evolution of conservation modules $G$ 'and loss $G$ ' 'as well as the evolution of the critical frequency with the concentration for a frequency range varying from 1 to $10 \mathrm{~Hz}$ and an imposed constraint of $1 \mathrm{~Pa}$, for concentrations [0.1\%-1\%].

For a concentration of $0.1 \%$, the curves below show the $\mathrm{G}$ 'which characterizes the conservation and the $\mathrm{G}$ ' 'which expresses the losses, we notice that the $\mathrm{G}^{\prime}$ 'is the dominant for a certain frequency range which is the Viscous behavior of the aqueous solution of the carbon nanotube with glycerol, the conservation factor prevails from a frequency of $4 \mathrm{~Hz}$ can close.

Hence the point of intersection between $G$ 'and $G^{\prime}$ 'at a critical value, $\mathrm{fc} \approx 4.601 \mathrm{~Hz}$

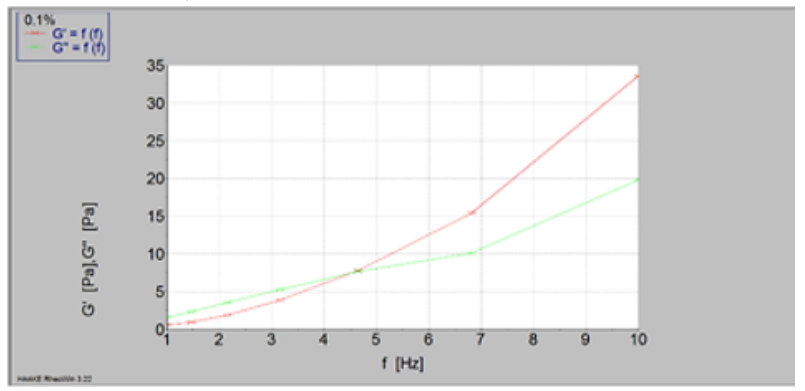

Figure 4: Viscoelastic properties of the solution for a concentration of $0.1 \%$.

A behavior similar to this one is observed for a concentration a concentration of $(0.2 \%-1 \%)$ where $\mathrm{f} \_\mathrm{c} \approx 6.335 \mathrm{~Hz}$ as shown in figure 2 .

It emerges from this analysis that the evolution of the value of fc, which corresponds to the case where $\mathrm{G}$ 'and $\mathrm{G}^{\prime}$ 'are comparable, is inversely proportional to the concentration. This consolidates the control protocols of aqueous solutions of carbon nanotubes with glycerol in oscillating mode and allows to optimize the processes of their use in an industrial environment.

The curve below shows the frequency as a function of the concentration these frequencies are taken for the loss module equal to the conservation module: 
It emerges from this analysis that the evolution of the value of $\mathrm{fc}$, which corresponds to the case where $\mathrm{G}$ 'and $\mathrm{G}^{\prime}$ 'are comparable, is inversely proportional to the concentration.

The curves below show the frequency as a function of the concentration. These frequencies are taken for the loss module equal to the conservation module:

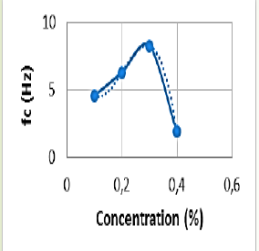

Figure5.a

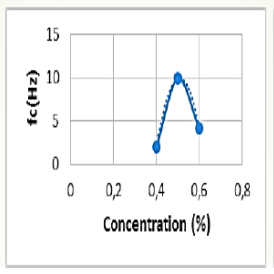

Figure5.b

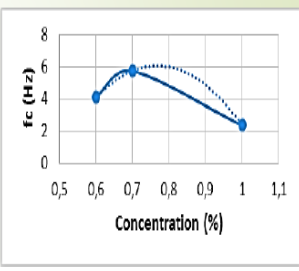

Figure5.c
Figure5: Curve shows the evolution of the frequency according to the concentration

a-For [0.1-0.4\%]

$\mathrm{fc}=-1411,2 \times 3+856,45 \times 2-140,81 \mathrm{x}+11,529$

b-For $[0.4-0.6 \%] \mathrm{fc}=-684,55 \times 2+695,28 \mathrm{x}-166,66$

c-For $[0,6-1 \%] \mathrm{fc}=-69,65 \times 2+107,11 \mathrm{x}-35,123$

This result is, in our opinion, an additional contribution to the control of the evolution of the flow of carbon nanotubes.

\section{Effect of temperature $\left[30-80^{\circ} \mathrm{C}\right]$ :}

We will take from RheoStress 1 a set of rheograms that show the effect of the temperature $\left[30-80^{\circ} \mathrm{C}\right]$ on the apparent

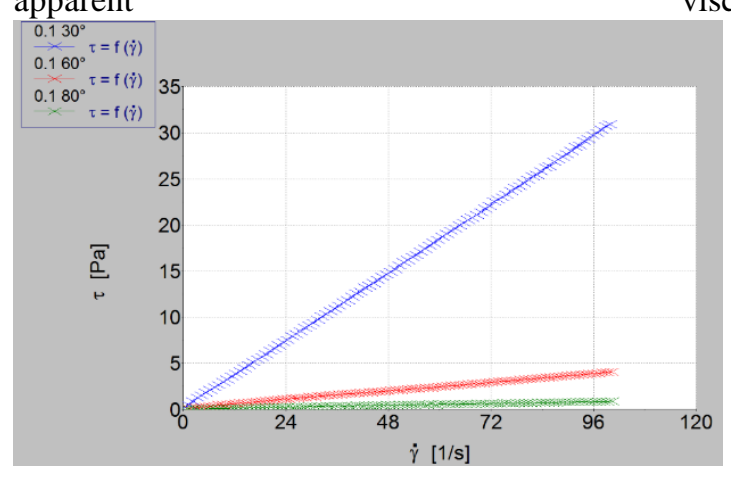

Fig. 6. Shear stress versus shear rate of carbon nanotube nanofluids-glycerol for different temperatures.

We studied the effect of temperature on the rheological behavior of the nanofluid. For this, we represent the evolution of the apparent viscosity as a function of temperature at different volume fractions (0.1-1\%)).

It is observed from Figures 2 that the apparent viscosity of the nanofluid decreases as the temperature increases in the temperature range studied (between 30 and $80^{\circ} \mathrm{C}$ ) and a constant shear rate.
Table1: effect of the parameters on the rheological and thermal behavior

\begin{tabular}{|c|c|c|c|c|}
\hline Viscosité & $\dot{y}(s-1)$ & $T{ }^{*} \mathrm{C}$ & $f m 96$ & Nanofluide \\
\hline 0,325 & 48,61 & 30 & & \\
\hline $\begin{array}{r}0,0475 \\
0,01114\end{array}$ & $\begin{array}{ll}48,65 \\
48,66 \\
\end{array}$ & 60 & $0,10 \%$ & Glycérol+NTC \\
\hline 0,557 & 48,59 & 30 & & \\
\hline 0,0706 & 48,58 & 60 & $0,20 \%$ & Glycérol+NTC \\
\hline 0,04814 & 48,59 & 80 & & \\
\hline 0,387 & 48,57 & 30 & & \\
\hline 0,02408 & 48,54 & 60 & $0,30 \%$ & Glycérol+NTC \\
\hline 0,0139 & $48,7 \mathrm{~s}$ & 80 & & \\
\hline 0,492 & 48,58 & 30 & & \\
\hline 0,483 & 48,47 & 60 & $0,40 \%$ & Glycérol+NTC \\
\hline 0,08673 & 48,6 & 80 & & \\
\hline 0,09182 & 48,59 & 30 & & \\
\hline 0,05178 & 48,47 & 60 & $0,50 \%$ & Glycérol+NTC \\
\hline 0,02779 & 48,6 & 80 & & \\
\hline 0,03512 & 48,49 & 30 & & \\
\hline 0,001578 & 48,66 & 60 & $0.60 \%$ & Glycérol+NTC \\
\hline 0,002212 & 48,71 & 80 & & \\
\hline 0.07885 & 48,5 & so & & \\
\hline 0,05436 & 48,59 & 60 & $0,70 \%$ & Glycérol+NTC \\
\hline 0,04844 & 48,59 & 80 & & \\
\hline 0,267 & 48,5 & 30 & & \\
\hline 0,293 & 48,59 & 60 & 1,0096 & Glyećral+NTC \\
\hline 0,383 & 48,59 & 80 & & \\
\hline
\end{tabular}

The temperature has a strong effect on the rheological properties of the CNT. The viscosity of NTC decreases significantly with increasing temperature.

\subsection{Thermal behavior:}

The thermal behavioral research was led by a heat exchanger to double envelope and it is due to its advantages such as the precise regulation of the temperature of the product with a homogeneous distribution of the temperatures.

We set as modus operandi, the fluid which circulates in the heating serpentine involves of the water with temperature $70^{\circ} \mathrm{C}$ for the heat transmission and for the reservoir, we took two cases for the cold fluid the first one involves some water and the 2nd the nanofluid with the nanotube of carbon and the glycerin the concentration between $0.3 \%$ and $0.7 \%$ of which we varied with a constant debit of $0.61 / \mathrm{min}$ with agitation.
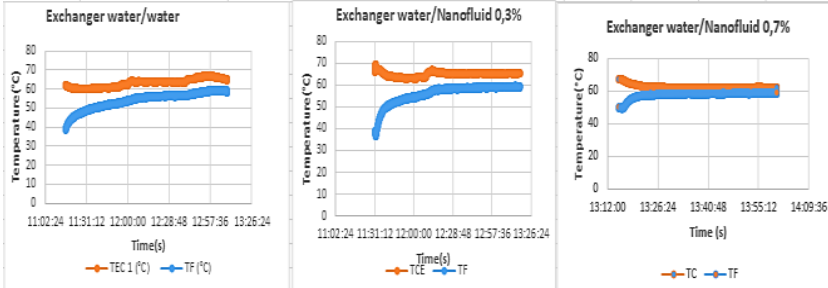

Figure.7: effect of the nature of the cold fluid in the tank on the heat exchange between TC and TF

It is noted that the heat exchange is affected by the nature of the cold fluid in the tank. It is observed that, when it is an exchange between the water and the nanofluid with $0.3 \%$ of carbon nanotube, the exchange is done quickly and the temperatures of the hot and cold fluid tend to the same value. This is confirmed in the case of the exchange between water and the nanofluid based on $0.7 \%$ of carbon nanotube. It should be noted that when the concentration of carbon nanotube increases the heat exchange increases.

\section{Références}

[1] Chen, L., Xie, H., Yu, W., \& Li, Y. (2011). Rheological behaviors of nanofluids containing multi-walled carbon nanotube. Journal of Dispersion Science and Technology, 32(4), 550554.

[2] Aladag B, Halelfadl S, Doner N, Mare T, Duret $\mathrm{S}$, Estelle P. Experimental investigations of the viscosity of nanofluids at low temperatures. Appl Energy 2012; 97:876-80 